nodules are undergoing diagnostic work up under the lung cancer multidisciplinary team (MDT) and 80 indeterminate nodules are under CT surveillance. The distribution of these cancers and nodules by eligibility criteria is shown in Table 1 .

Abstract S14 Table 1 Number of cancers and nodules by eligibility criteria *NLST criteria but with modified age range of 60 to 75 years

\begin{tabular}{lllll}
\hline & $\begin{array}{l}\text { PLCO }_{\text {m2012 }} \\
\text { positive }\end{array}$ & $\begin{array}{l}\text { LLP } \\
\text { positive }\end{array}$ & $\begin{array}{l}\text { NLST-like* } \\
\text { positive }\end{array}$ & $\begin{array}{l}\text { Total in } \\
\text { cohort }\end{array}$ \\
\hline Had CT & 576 & 661 & 493 & 718 \\
$\begin{array}{l}\text { Indeterminate nodules } \\
\text { Suspicious nodule referred }\end{array}$ & 64 & 74 & 58 & 80 \\
to MDT & 8 & 9 & 7 & 10 \\
\begin{tabular}{l} 
Confirmed cancers \\
\hline
\end{tabular} & 17 & 16 & 13 & 17 \\
\hline
\end{tabular}

Conclusions Using the NLST-like* criteria to determine eligibility would mean the fewest number screened, with 4 fewer cancers detected. The $\mathrm{PLCO}_{\mathrm{m} 2012}$ score was the most reliable way to detect cancers and resulted in less individuals screened than with use of the LLP score. Further follow up and review of the data is required to fully establish the most effective tool for determining eligibility into LDCT screening though the PLCO $_{\mathrm{m} 2012}$ score shows the most promise with the available data.

\section{S15 IMPROVING THE RISK STRATIFICATION FOR MALIGNANCY IN SMALL PULMONARY NODULES FROM AN UNSELECTED PATIENT POPULATION}

${ }^{1} \mathrm{~A}$ Talwar, ${ }^{2} \mathrm{JMY}$ Willaime, ${ }^{1} \mathrm{~N}$ Rahman, ${ }^{2} \mathrm{M}$ Gooding, ${ }^{3} \mathrm{~T}$ Kadir, ${ }^{1} \mathrm{~F}$ Gleeson. ${ }^{1} \mathrm{Oxford}$ University Hospitals NHS Foundation Trust, Oxford, UK; ${ }^{2}$ Mirada Medical Ltd, Oxford, UK; ${ }^{3}$ Optellum Ltd, Oxford, UK

10.1136/thoraxinl-2017-210983.21
Introduction Distinguishing between benign and malignant small pulmonary nodules (PNs) detected on CT scanning is a significant challenge. Such nodules are commonly detected in clinical practice as incidental findings or in patients with a history of prior malignancy. CT texture analysis (CTTA) has been proposed as a potential imaging biomarker in tumour characterisation. Image texture refers to the statistical analysis of spatial intensity variations of the pixels within an image to produce a CT texture score. This score is then mapped onto a probability of malignancy from $0-1$.

Aims and Objectives

- To create a registry of patients with small solid PNs from an unselected population of patients.

- Patient demographic data were combined with information acquired from CT derived parameters such as shape, size, and texture analysis (CTTA) to develop and validate a generalised linear model to determine the probability of malignancy of PNs.

- A parallel prospective interventional cohort study was also conducted to assess whether CTTA repeatability was comparable to automatic volumetric measurements when a patient is scanned twice on the same day.

Methods Between January 2012 to September 2014, 1008 patients presenting with small solid PNs were identified. The gold standard diagnosis of the nodules was established by histology or nodule stability at 2 years of CT follow-up.

Results The prevalence of malignant PNs was 31.6\% (319/ 1008). Significant independent predictors of malignancy included prior history of malignancy within 5 years $(\mathrm{OR}=117.4,(95 \%$ confidence interval(CI)):67.1 to 272.8, $\mathrm{p}<0.001)$; larger nodule diameter $(\mathrm{OR}=9.7, \mathrm{CI}: 4.1$ to 17.6 , $\mathrm{p}<0.001)$; nodule count $(\mathrm{OR}=1.6, \mathrm{CI}: 1.3$ to $1.8, \mathrm{p}<0.001)$ and nodule spiculation $(\mathrm{OR}=118.4, \mathrm{CI}: 61.9$ to 772.3 , $\mathrm{p}<0.001)$. The models' performance using the area under the ROC curve (AUC) was 0.969. When CTTA was used alone the AUC was 0.800 (figure 1). CTTA displayed ULR and LLR

Abstract S15 Figure 1 (A) Patient demographics and nodule characteristics, (B) Performance of clinical models (AUC is area under the ROC curve), and (C) Bland-altman plot to show variability in texture feature scores and volumetry for 40 Pulmonary nodules. 
below $\pm 17.8 \%$, comparable to volume using Bland-Altman and also had high repeatability $\{$ CCC $(0.84 \leq \mathrm{CCC} \leq 0.99)\}$.

Conclusion This study has highlighted the potential clinical utility of CTTA in the risk stratification of PNs. It has also shown that CTTA is a highly repeatable imaging biomarker of malignancy, akin to volume measurements but with the advantage of not requiring imaging follow-up.

\section{Understanding and treating those irritating infections}

\section{S16 CIRCADIAN CONTROL OF PRIMARY LUNG ALLOGRAFT DYSFUNCTION, MEDIATED BY THE CLOCK PROTEIN, REVERB $\alpha$}

${ }^{1} \mathrm{PS}$ Cunningham, ${ }^{1} \mathrm{HJ}$ Durrington, ${ }^{2} \mathrm{RV}$ Venkateswaran, ${ }^{3} \mathrm{M}$ Cypel, ${ }^{3} \mathrm{~S}$ Keshavjee, ${ }^{1} \mathrm{JE}$ Gibbs, ${ }^{1}$ AS Loudon, ${ }^{3} \mathrm{CW}$ Chow, 'DW Ray, ' JF Blaikley. ${ }^{1}$ The University of Manchester, Manchester, UK; ${ }^{2}$ University Hospitals of South Manchester, Manchester, UK; ${ }^{3}$ Toronto General Hospital, Toronto, Canada

\subsection{6/thoraxjnl-2017-210983.22}

Introduction The circadian clock regulates murine immune responses by time of day, partly through the clock protein REVERB $\alpha$, resulting in altered mortality after infection. The mechanisms regulating time of day differences are poorly understood in humans, where performing circadian studies presents a number of challenges. Lung transplantation, which is performed at any time of day to minimise organ ischaemic time, is an ideal model to study circadian effects on human immune responses.

Methods Primary graft dysfunction (PGD) incidence after lung transplantation was examined for an eight year retrospective (2004-2012) cohort $(n=563)$ in one centre. Patients were excluded, a priori, if they had significant intra-operative complications, had a previous lung transplant, or if the donor lung had undergone ex-vivo perfusion. Circadian factors were also studied using PER2::Luc and REVERB $\alpha^{-/-}$mice and by pharmacological targeting of the circadian clock in primary alveolar macrophages from lung transplant recipients.

Results The incidence of PGD grades $2 / 3$ at 24 hours was temporarily elevated when organs were reperfused between 4 and 8 am $(\mathrm{p}<0.02)$ compared to other time points. Similar observations were made when the cohort was examined by operation start time $(\mathrm{p}<0.01)$. Sub-cohort analysis, defined using ISHLT relative contraindications, revealed that PGD incidence oscillated in a circadian manner $\left(r^{2}=0.87, p=0.046\right)$. Investigations in PER2::Luc mice, which allows real time tracking of circadian oscillations, revealed that temperature and serum fluctuations, mimicking organ preservation, shifts the donor organ clock by 4-12 hours depending on time of retrieval. This could create circadian desynchrony between the transplanted organ and recipient. In macrophages, genomewide gene expression analysis of the role of REVERB $\alpha$ identified gene ontology terms linked to the regulation of lymphocyte function and activation, suggesting a functional link from the macrophage to the adaptive immune response. Furthermore, key PGD biomarkers are elevated $(\mathrm{p}<0.05)$ in macrophages from REVERB $\alpha^{-/}$mice and are repressed $(\mathrm{p}<0.05)$ by REVERB ligands (GSK2945 and GSK2667) in macrophages from lung transplant recipients.
Conclusion This study suggests that the circadian clock could temporarily affect outcomes after lung transplantation due to recipient-donor circadian desynchrony. Ligands targeting the clock protein REVERB $\alpha$ repress key PGD biomarkers showing that this is a tractable therapeutic pathway.

\section{S17 LATENT CLASS MODELLING FOR PULMONARY ASPERGILLOSIS DIAGNOSIS IN LUNG TRANSPLANT RECIPIENTS}

${ }^{1} \mathrm{~A}$ Shah, ${ }^{2} \mathrm{~A}$ Abdolrasouli, ${ }^{1} \mathrm{~S}$ Schelenz, ${ }^{3} \mathrm{C}$ Thornton, ${ }^{2} \mathrm{MZ} \mathrm{Ni},{ }^{1} \mathrm{~A}$ Devaraj, ${ }^{1} \mathrm{~N}$ Devic ${ }^{1} \mathrm{~L}$ Ward, ${ }^{1} \mathrm{M}$ Carby, ${ }^{1} \mathrm{~A}$ Reed, ${ }^{2} \mathrm{C}$ Costelloe, ${ }^{2} \mathrm{D}$ Armstrong-James. ${ }^{1}$ Royal Brompton and Harefield NHS Foundation Trust, London, UK; ${ }^{2}$ Imperial College London, London, UK; ${ }^{3}$ University of Exeter, Exeter, UK

\subsection{6/thoraxjnl-2017-210983.23}

Rationale Timely, accurate diagnosis of invasive aspergillosis (IA) is key to enable initiation of antifungal therapy in lung transplantation. Despite promising novel fungal biomarkers, the lack of a diagnostic gold-standard creates difficulty in determining utility.

Objectives This study aimed to use latent class modelling of fungal diagnostics to classify lung transplant recipients (LTR) with IA in a large single centre.

Methods Regression models were used to compare composite biomarker testing of bronchoalveolar lavage to clinical and EORTC-MSG guideline-based diagnosis of IA with mortality used as a surrogate primary outcome measure. Bootstrap analysis identified radiological features associated with IA. Bayesian latent class modelling was used to define IA.

Measurements and Main Results A clinical diagnosis of fungal infection $(P=<0.001)$ and composite biomarker positive Results $(P=<0.001)$ had significantly increased 12 month mortality. There was poor correlation between clinical diagnosis, EORTC-based IA diagnosis and composite biomarker positivity. Tracheobronchitis was positively predictive of a clinical and composite biomarker positive diagnosis of IA $(\mathrm{p}=0.004 ; 95 \%$ CI-1.79-21.28 and $\mathrm{p}=0.03 ; 95 \% \mathrm{CI}-0.85-$ 15.62 respectively). Latent class modelling resulted in the formation of 3 groups: Class 1: likely fungal infection; Class 2: unlikely fungal infection; Class 3: unclassifiable. A. fumigatus PCR was positive in $\sim 90 \%$ of class 1 LTRs compared to only $1 \%$ in class 2. Analysis of mortality showed a trend towards significance comparing class 1 with class 2 ( $\mathrm{p}=0.06$; HR4.7;95\% CI(0.91-24)) (figure 1).

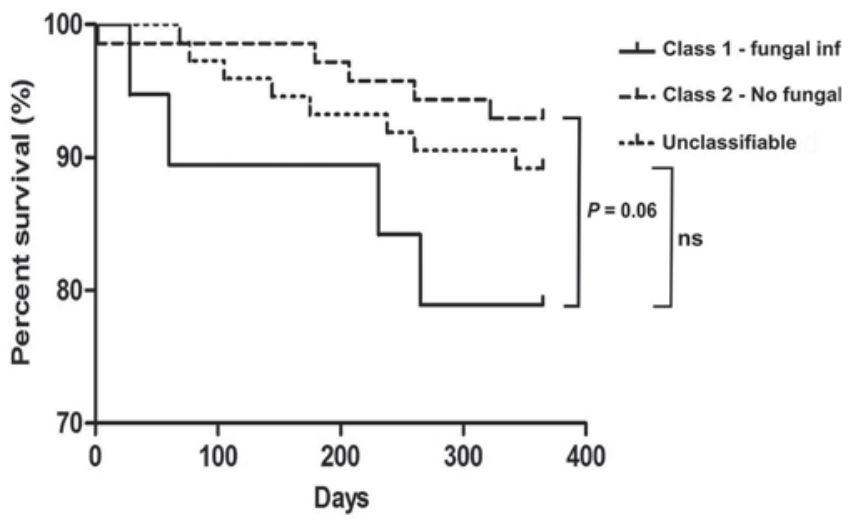

Abstract S17 Figure 1 